\section{Dual anti-platelet therapy and dento-alveolar surgery. How do we manage patients on anti-platelet medication?}

\author{
N. Patel, ${ }^{1}$ V. Patel, ${ }^{2}$ D. Sarkar, ${ }^{3}$ T. Nokes ${ }^{4}$ and P. Blacklock ${ }^{5}$
}

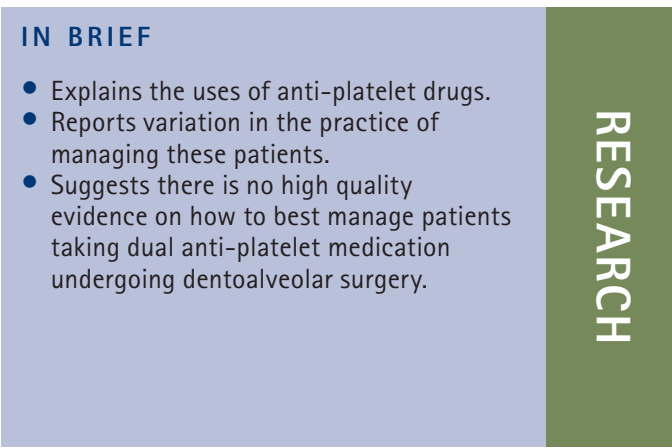

\begin{abstract}
Objective The optimum management of dual anti-platelet therapy (DAPT) in patients undergoing dentoalveolar surgery is controversial. The lack of guidelines has meant clinicians have been left to manage these cases from specialist advice or past experience. In view of this the British Association of Oral Surgeons (BAOS) membership was surveyed to assess current practice. Design An electronic survey consisting of nine closed multiple-choice questions was circulated to all 435 registered BAOS members. A section for open comments was also included. The data was transcribed, organised and reduced to themes by hand and finally analysed. Results One hundred and eighty members completed the questionnaire giving a response rate of $41 \%$. Ninety-two percent of clinicians had direct involvement in managing patients on DAPT and of these $80 \%$ had not encountered a bleeding complication. DAPT was continued for simple dental extraction under local anaesthetic by $83 \%$ of respondents and $73 \%$ for surgical extractions. Two thirds of the respondents did not follow any guidelines but for those that did the most common was the UK Medicines Information protocols. Only 10\% of respondents reported a significant post-operative bleeding complication following surgery in patients on DAPT. Conclusion This survey confirmed the continued variable management of patients on DAPT undergoing dentoalveolar surgery. Reported bleeding rates were low with over 75\% of patients not having their DAPT treatment interrupted.
\end{abstract}

\section{INTRODUCTION}

The incidence of an ageing population living with complex medical and drug histories is well documented. ${ }^{1}$ There are many medical problems facing the ageing population with cardio- and cerebrovascular disease being among the most common. A recent paper by Patel et $a .^{2}$ demonstrated over a third of patients seeking emergency dental treatment had a medical co-morbidity, the most common being cardiovascular disorders which are frequently managed with anticoagulants and/or anti-platelet medication. Management of these patients provides a challenge for the dentist, which is set to continue with improvement in oral health, access to dentistry and the ability to provide advanced restorative treatments leading to retention of teeth for longer.

${ }^{1}$ Academic Clinical Fellow in Oral Surgery, The University of Manchester, School of Dentistry, Coupland 3 Building, Coupland Street, Manchester, M13 9PL; ${ }^{2}$ StR Oral Surgery, Fl 23, Oral Surgery Department, Guys Dental Hospital, Guys \&t St Thomas NHS Foundation Trust, London Bridge, London, SE1 9RT; ${ }^{3}$ Cons Cardiologist, ${ }^{4}$ Consultant Haematologist, ${ }^{5}$ Associate Specialist Oral and Maxillofacial Surgery, Derriford Hospital, Plymouth, PL6 8DH

${ }^{*}$ Correspondence to: Neil Patel

Email: Neil.Patel@cmft.nhs.uk

Online article number E24

Refereed Paper - accepted 20 October 2014

DOI: 10.1038/sj.bdj.2014.1055

${ }^{\circ}$ British Dental Journal 2014; 217: E24

\begin{tabular}{|c|c|c|c|c|c|}
\hline & Aspirin (ASA) & + Clopidogrel & + Prasugrel & + Ticagrelor & + Dipyridamole SR \\
\hline Target & $\cos 1$ & ADP receptor & ADP receptor & ADP receptor & $\begin{array}{l}\text { Inhibits platelet } \\
\text { uptake of adenosine }\end{array}$ \\
\hline Mode & Irreversible & Irreversible & Irreversible & Reversible & Reversible \\
\hline Indications & $\begin{array}{l}1^{\circ} \text { and } 2^{\circ} \\
\text { prevention of } \\
\text { atherothrombosis } \\
\text { ACS, stroke and } \\
\text { PVD }\end{array}$ & $\begin{array}{l}\text { ACS, } 2^{\circ} \text { prevention } \\
\text { of stroke, stented } \\
\text { peripheral and } \\
\text { coronary artery } \\
\text { disease }\end{array}$ & $\begin{array}{l}\text { ACS patients } \\
\text { treated with } \\
\text { a coronary } \\
\text { stent }\end{array}$ & $\begin{array}{l}\text { All ACS } \\
\text { patients }\end{array}$ & $\begin{array}{l}2^{\circ} \text { prevention } \\
\text { of stroke }\end{array}$ \\
\hline $\begin{array}{l}\text { Platelet } \\
\text { inhibition }\end{array}$ & Weak & Moderate & High & High & Moderate \\
\hline $\begin{array}{l}\text { Interval for } \\
\text { platelet } \\
\text { recovery }\end{array}$ & 5 days & 57 days & 57 days & $\begin{array}{l}2 \text { days } \\
\text { ( } 5 \text { days in } \\
\text { drug licence) }\end{array}$ & 2 days \\
\hline
\end{tabular}

A range of oral anti-platelet drugs are available for managing conditions associated with the cardio- and cerebrovascular systems with aspirin, clopidogrel and dipyridamole commonly encountered (Table 1). Aspirin and clopidogrel can be used both individually and in combination as dual anti-platelet therapy (DAPT). Aspirin prevents platelet aggregation and subsequent thrombus formation by irreversibly inhibiting platelet cyclo-oxygenase-1(COX-1) thus preventing the formation of thromboxane A2, a potent vasoconstrictor and platelet aggregant. ${ }^{3}$ Aspirin has an effect on platelet function but not platelet count. ${ }^{4}$ Clopidogrel irreversibly inhibits platelet activity by disrupting aggregation through an antagonistic effect at the ADP receptor PY12. ${ }^{5}$ Clopidogrel is considered a more potent anti-platelet agent and can prolong the bleeding time by 1.5 to 3 times normal. ${ }^{6}$ Dipyridamole is an adenosine reuptake inhibitor and phosphodiesterase inhibitor with anti-platelet and vasodilating activity. ${ }^{7}$ The action of dipyridamole on platelets is reversible and detectable within an hour of ingestion. Clopidogrel 
has a peak effect 2-3 hours after ingestion with the ADP receptors irreversibly blocked for the life of the platelets (7-10 days). ${ }^{8,9}$

DAPT is widely used following an acute coronary syndrome (ACS), ${ }^{10,11}$ percutaneous coronary intervention (PCI), symptomatic peripheral vascular disease with percutaneous lower extremity revascularisation ${ }^{12}$ as well as for the prevention of recurrent stroke. ${ }^{13}$ The combination of aspirin with clopidogrel has been shown to reduce death, non-fatal MI and stroke following an ACS event with the major effect being in the first six months. Recurrent vascular events are reduced by about a quarter with the use of DAPT compared with aspirin alone. ${ }^{10}$

The Myocardial Ischaemia National Audit Project (MINAP) ${ }^{14}$ has documented the reductions in mortality resulting from changes in acute treatment of MI and the application of secondary prevention measures. ${ }^{15}$ The NICE guidelines on secondary prevention of MI (2013) recommends that those who have suffered an MI should be offered dual anti-platelet therapy for 12 months. ${ }^{15}$ NICE's recommendation for its use will mean that dentists will continue to see patients presenting on DAPT.

The in vitro response to clopidogrel shows a wide variation between individuals. ${ }^{16}$ This reflects a two-stage enzymatic degradation required to produce the active clopidogrel metabolite. Variation in the activity of liver enzymes particularly CYP2C19 accounts for this. ${ }^{16} \mathrm{New}$ anti-platelet agents with a more consistent and often higher degree of platelet inhibition are now available. These new $\mathrm{ADP}$ receptor blockers become active with a single metabolic step (prasugrel) or require no metabolism at all (ticagrelor). Their use is currently restricted to the ACS population (ticagrelor) or stented ACS patients (prasugrel). Further anti-platelet agents may soon be launched in the UK including elinogrel and cangrelor. Both ticagrelor and prasugrel have been subject to a NICE technology appraisal (TA 236 Oct 2011, ${ }^{17}$ TA 182 Sept $2009^{18}$ ) and accepted as alternatives to clopidogrel. Therefore these agents will become more widely encountered in dental practice. For the moment the combination of aspirin and clopidogrel remains the commonest form of DAPT.

DAPT is associated with an increased risk of bleeding. Premature discontinuation of DAPT following an ACS or coronary stent implantation is also associated with an increased risk of a recurrent coronary event or stent thrombosis. Therefore the decision to discontinue treatment, even temporarily, is often finely balanced. The current article concentrates on the commonest DAPT combination of aspirin/clopidogrel and the oral surgeon's experience of its effects on the outcomes of dentoalveolar surgery.

Guidelines and evidence for the management of patients on DAPT and dentoalveolar surgery remains limited. This has resulted in uncertainty about what constitutes best practice. In view of this sparsity of information, the authors surveyed the British Association of Oral Surgery (BAOS) membership to identify current practice and reviewed these responses in the context of current published literature.

\section{METHODS}

A survey consisting of nine closed multiple choice questions was developed and piloted by the authors. The questions were directed to enquire about individual clinician's management of patients taking aspirin and clopidogrel while undergoing dentoalveolar surgery procedures. (Table 2). A section for open comments was available for all questions. The study was carried out under the auspices of the British Association of Oral Surgeons and participation was voluntary. Once piloted it was transcribed onto an online platform and emailed to all 442 registered members of the British Association of Oral Surgeons who had provided an email address. Participants were informed that responses were anonymised. A reminder email was sent after four weeks to those who had not completed the initial survey. Survey results were collected over a 20 week period. Quantitative data was checked, coded and entered by two people to reduce error. SPSS for Windows package version 19 (SPSS: An IBM Company Chicago, IL, USA) was used for data analysis. Response rates were recorded to review any risk of response bias and missing data noted. Open questions responses were categorised using a thematic approach. ${ }^{19}$ The data was transcribed, organised and then reduced to themes by hand and analysed. Themes were reviewed independently by two people to ensure accordance of themes and to increase the validity of the findings.

\section{RESULTS}

Of the 442 emailed questionnaires sent, seven member's emails were invalid. From the 435 successfully sent questionnaires, 212 members viewed the survey but did not respond and 180 were successfully completed giving a response rate of $41 \%$. All questions were fully answered except questions 3 and 7 which had a 92\% completion rate. Four questions had additional comments.

Ninety-two percent of BAOS members, responding to the questionnaire, were involved directly in the management of patients prescribed DAPT (aspirin and clopidogrel) while needing to undergo a

\section{Table 2 Questionnaire}

\section{Questions}

Have you been involved in the management of patients on dual anti-platelet agents aspirin/ clopidogrel undergoing dentoalveolar surgery.

Do you have guidelines to follow for the management of these patients? Please describe the guidelines/method 'you' follow

How is their anti-platelet treatment managed before surgery?

In general, what precautions do you take for a simple extraction under local anaesthetic?

In general, what precautions do you take for a surgical extraction under local anaesthetic?

What measures do you use to obtain haemostasis?

Do you have a different protocol for managing a patient on both aspirin \& clopidogrel if they are undergoing a general anaesthetic for their dentoalveolar surgery?

Have you experienced any bleeding complication in patients who were on both aspirin \& clopidogrel while undergoing dentoalveolar surgery?

Where is the majority of your oral surgery work carried out?

dentoalveolar surgical procedure. Of these, 80\% had not encountered any bleeding complications while treating patients on dual aspirin and clopidogrel. The respondents worked in a number of different environments; 52\% were based in district general hospitals, 19\% in dental hospitals, 15\% in specialist practice, $4 \%$ in general dental practice and 6\% in salaried dental services.

When asked if they followed any guidelines for the management of patients taking aspirin and clopidogrel, 66\% did not follow any guidance or did not answer the question (Fig. 1). Only 58\% of respondents liaised with the patient's general practitioner or cardiologist when planning dentoalveolar surgery. The majority of respondents continued both aspirin and clopidogrel for simple dental extractions under local anaesthetic (83\%), for surgical extractions under local anaesthetic (73\%) and treatment under general anaesthetic (87\%) (Figs 2 and 3). The most popular local measures used to obtain haemostasis following the surgical procedure were sutures (29\%) and local haemostatic dressings (28\%).

Open responses suggested that the most commonly used national guidelines for patients on aspirin and clopidogrel undergoing dentoalveolar procedures were the UKMi (North West) protocols. ${ }^{20}$ Forty percent of those questioned quoted low levels of evidence for the guidance being used to inform their clinical decisions. This included their own experience, lectures from consultants, and liaison with departmental colleagues. 
When further advice was sought regarding management, oral surgeons liaised most commonly with cardiologists, the patient's general practitioner and haematologists. The majority of those questioned did not feel it was their role or appropriate for the oral surgeon to modify the dose or ask the patient to omit their anti-platelet medication independently. However, those practitioners that did request changes in dual anti-platelet medication before surgery usually preferred their patient to stop taking clopidogrel up to 14 days before the surgery. No practitioner stated they would discontinue the aspirin.

Responders were further asked to comment whether they used any additional measures to promote haemostasis or prevent bleeding in patients on aspirin and clopidogrel. Responses ranged from some practitioners using no additional measures, reducing the number of teeth extracted per episode or using sutures and placing a haemostatic dressing such as Surgicel ${ }^{\circledR}$ in the sockets following extraction. The importance of delivering good quality post-operative instructions to the patients was a common theme cited by respondents to the open question. Practitioners felt it was important that detailed instructions were given to the patient including what to do if bleeding occurred at home following the extraction.

The number of reported complications following the management of patients on dual anti-platelet therapy was low. Only $10 \%$ of respondents reported a significant post-operative bleeding complication following dentoalveolar surgery in patients on aspirin and clopidogrel. Open responses suggested that oral surgeons noticed increased bleeding in this patient group at the time of surgery this being managed by limiting the number of extractions in one visit and using local measures.

\section{DISCUSSION}

The major role of anti-platelet drugs is to prevent thrombosis in atherosclerotic arteries leading to major complications such as myocardial infarction, stroke and critical limb ischaemia. ${ }^{5}$ All anti-platelet medications affect clotting by inhibiting platelet aggregation but they do so by a variety of different mechanisms. ${ }^{8}$ There are a number of drugs under the classification of antiplatelets and these include aspirin, other NSAIDs, clopidogrel, dipyridamole and fibrinogen receptor inhibitors.

The term DAPT generally encompasses the use of aspirin and clopidogrel together. Evidence has shown their action to be additive and indications for its use include:

- Acute coronary syndrome without ST segment elevation

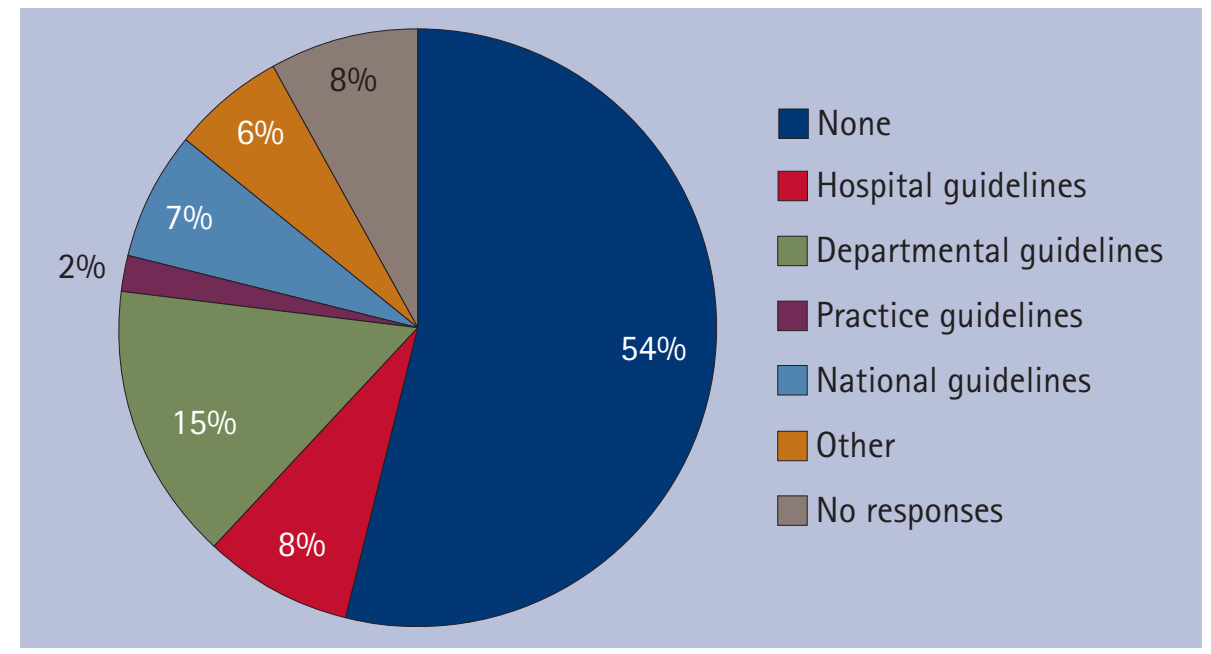

Fig. 1 Chart to show frequency of guidelines followed, $n=180$

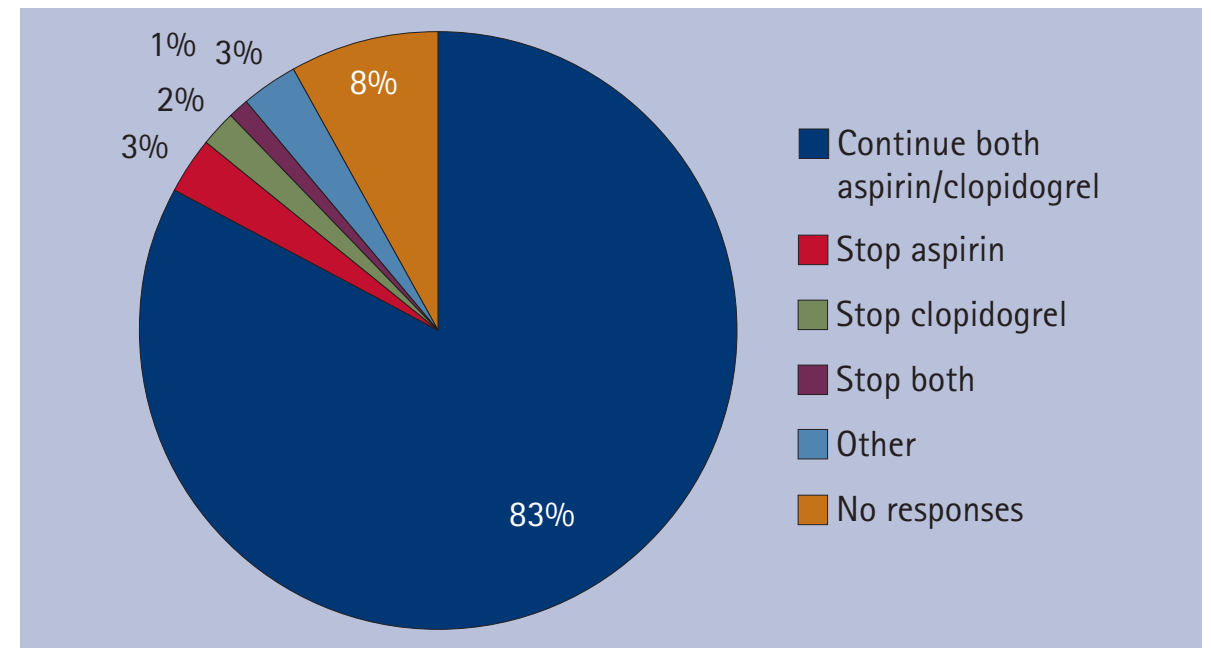

Fig. 2 Chart to show management of patients on DAPT undergoing simple dental extractions under local anaesthetic, $n=180$

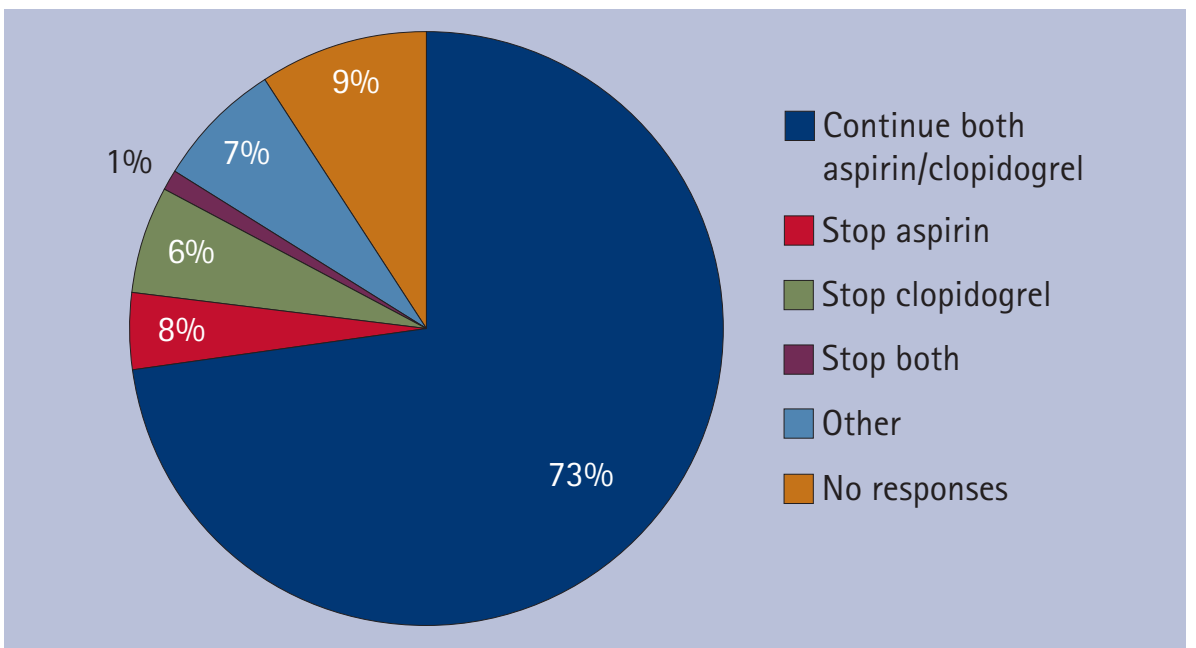

Fig. 3 Chart to show management of patients on DAPT undergoing simple dental extractions under local anaesthetic, $n=180$

- Acute myocardial infarction with ST segment elevation

- Patients undergoing percutaneous coronary intervention: coronary artery stents (bare metal stents, drug eluting stents insertion

- Prevention of atherothrombotic and thromboembolic events in patient with atrial fibrillation (and at least one risk

factor for a vascular event)

- Where warfarin is unsuitable.

The major concern for patients being treated with dual anti-platelet therapy is a 
peri- or post-operative bleeding complication. Clinically significant post-operative bleeding following dental procedures has been defined as that which:

- Continues beyond 12 hours ${ }^{21}$

- Causes the patient to call or return to the dental practice or accident and emergency department ${ }^{21}$

- Results in the development of a large haematoma or ecchymosis within the oral soft tissues ${ }^{21}$

- Requires a blood transfusion. ${ }^{21}$

The complication of bleeding, however, needs to be balanced against the risk or recurrent atherothrombotic event associated with withdrawl of one or both antiplatelets before dental surgery. A guidance document produced by six American stakeholder societies recommended that dual anti-platelet therapy should be uninterrupted in patients with drug-eluting stents who undergo dental procedures..$^{22}$ It was recognised that even though this recommendation had been made, prospective studies specifically assessing the safety of this approach are lacking. This was echoed by UKMi review which concluded there is insufficient evidence to comment on the bleeding risk if patients take both aspirin and clopidogrel. ${ }^{20}$

A more recent prospective study by Lillis et $a l .{ }^{23}$ investigated dental extraction during uninterrupted single or DAPT. The prospective study divided patients into four groups: aspirin alone; clopidogrel alone; aspirin and clopidogrel; and a control group on no antiplatelet therapy. They found, compared to the control group, the dual therapy patients had an increase tendency for immediate postoperative bleeding but this could be simply managed by local haemostatic measures (suturing and application of oxidised cellulose gauze). This increase in immediate postoperative bleeding did not progress towards a higher incidence of late haemorrhage. They therefore concluded that dental extractions may be safely performed in patients receiving single or dual anti-platelet therapy when appropriate local haemostatic measures are taken, thus averting the thrombotic risk of interrupting anti-platelet therapy.

Napeñas et al..$^{24}$ retrospective study reported on 43 patients on anti-platelet therapy (29 dual therapy) undergoing invasive procedures with no documented episodes of post-operative bleeding. The authors' conclusion was consistent with that of Lillis et al., ${ }^{23}$ however, this study had limitations due to its retrospective approach as well as the small number of subjects

As stated, the evidence surrounding the oral surgery management of DAPT remains limited. On the contrary there is an abundance of evidence and guidelines on the safety and suggested management approach for patients on warfarin or aspirin mono-therapy. They advise that anti-platelet medications should only be discontinued in the perioperative period when the haemorrhagic risk of continuing them is definitely greater than the cardiovascular risk associated with their discontinuation. This advice has been strengthened by the conclusions of recently published reviews ${ }^{6,25,26}$ and large cohort studies. ${ }^{27}$ Furthermore, the American Academy of Neurology recommends that patients undergoing dental procedures continue taking aspirin or warfarin for stroke prevention.

The responses to our questionnaire survey shows lack of consistency in the management of DAPT patients undergoing dentoalveolar surgery. This reflects the current lack of evidence and clear guidance provided to clinicians in the management of patients on DAPT.

As with all questionnaire surveys of this nature there may be an element of responder bias producing results that are not truly reflective of the wider current practice of dentoalveolar procedures in the context of DAPT. Our sample size was adequate with an acceptable response rate of $41 \%$ with patients managed in both the primary and secondary care setting. Selecting the sample from a population of BAOS members may add a further source of bias in that this group may have additional training and understanding of the management of this group of patients. General dental practitioners who are treating these patients on a daily basis in a primary setting have little national guidance at present and less easily available access to cardiological or haematological advice.

The majority of responders to the survey stated that they would not alter the patient's anti-platelet medication but instead would use additional measures to ensure haemostatsis. This finding further emphasises the holistic management of patients by oral surgeons, and their ability to recognise the importance of the medication taken by their patients. It was also encouraging to see oral surgeons collaborating with their medical colleagues in the management of patients on DAPT.

The majority of those oral surgeons questioned worked in secondary care units and therefore may be well rehearsed in the management of these patients. They would also have ready access to senior cover and specialist equipment/materials. It would be interesting to note how many of the patients treated were referred from general dental practitioners, due to their discomfort at managing these patients in a primary setting. The
UKMi review ${ }^{20}$ concluded there is insufficient evidence to comment on the bleeding risk if patients take both aspirin and clopidogrel. These patients may need to be referred to a dental hospital or hospital-based oral/maxillofacial surgeon. ${ }^{20,28,29}$ Once again the formulation of clear guidance would assist in the risk assessment of these patients and state which patients would benefit from management in secondary care.

A mixed response was given when we questioned what guidance the surgeons referred to when managing these patients. A number of 'guidelines' were quoted all with varying levels of evidence base. Guidelines varied from national statements based on clinical trials to 'expert' opinion from their senior clinician. It is important that clinicians are able to appraise the evidence used to formulate the guidelines they currently follow to ensure their practice is up-to-date.

This survey has demonstrated the lack of current evidence and guidance available to oral surgeons and general dental practitioners alike for the management of patients taking DAPT requiring dentoalveolar surgical procedures. With the ever increasing number of patients taking DAPT and the introduction of novel and potentially more potent anti-platelet agents (ticagrelor/prasugrel) to be used in DAPT there is a pressing need for new evidence-based guidelines to be produced.

The authors would like to thank the British Association of Oral Surgeons membership for their contribution to the survey.

1. Nobili A, Garattini S, Mannucci P M. Multiple diseases and polypharmacy in the elderly: challenges for the internist of the third millennium $J$ Comorbidity 2011; 1 : 28-44.

2. Patel, N, Broadfield, L J, Mellor A C. Medical profile of patients accessing hospital-based emergency dental care. Oral Surg. 2013; 7: 26-32.

3. Ferrari $E$, Benhamou M, Cerboni $P$, Marcel B. Coronary syndromes following aspirin withdrawal: a special risk for late stent thrombosis. J Am Coll Cardiol 2005; 45: 456-459.

4. George J N, Shattil S J. The clinical importance of acquired abnormalities of platelet function. $N$ Engl J Med 1991; 324: 27-39.

5. Nizarali N, Rafique S. Special care dentistry: Part 2. Dental management of patients with drug-related acquired bleeding disorders. Dent Update 2013; 40: 711-771.

6. Lecompte T, Hardy J-F. Antiplatelet agents and perioperative bleeding. Can J Anesth 2006; 53: S103-S112.

7. Dipyridamole. In Sweetman S C (ed). Martindale: The complete drug reference. 34th ed (electronic version). London: Pharmaceutical Press, 2005. Available online via www.medicinescomplete.com (accessed 7 July 2014).

8. Merritt J C, Bhatt D L. The efficacy and safety of perioperative anti-platelet therapy. J Thromb Thrombolysis 2002; 13: 97-103.

9. Clopidogrel (Drug Evauation) Klasco RK (Ed): DRUGDEX ${ }^{\circledR}$ System (electronic version). Thomson Micromedex, Greenwood Village, Colorado U S A. Information available online at http://www.app. dundee.ac.uk/tuith/Static/info/antiplatelet.pdf. (accessed 21 November 2014).

10. The Clopidogrel in Unstable Angina to Prevent Recurrent Events Trial Investigators. Effects of 
clopidogrel in addition to aspirin in patients with acute coronary syndromes without ST-segment elevation. N Eng/ J Med 2001; 345: 494-502.

11. COMMIT (Clopidogrel and Metoprolol in Myocardial Infarction Trial) collaborative group. Addition of clopidogrel to aspirin in 45,852 patients with acute myocardial infarction: randomised placebo-controlled trial. The Lancet 2005; 366: 1607-1621.

12. Gouya G, Arrich J, Wolzt M et al. Antiplatelet treatment for prevention of cerebrovascular events in patients with vascular diseases: a systematic review and meta-analysis. Stroke 2014; 45: 492-503.

13. Hanna E B. Dual anti-platelet therapy in peripheral arterial disease and after peripheral percutaneous revascularization. J Invasive Cardio/ 2012; 24: 679-684.

14. Myocardial Ischaemia National Audit Project. Information available online at http://www.ucl.ac.uk/ nicor/audits/minap (accessed 5 November 2014).

15. Ml-secondary prevention. Secondary prevention in primary and secondary care for patients following a myocardial infarction. NICE clinical guideline 172. November 2013. Guideline available online at http://www.nice.org.uk/guidance/cg172 (accessed 5 November 2014).

16. Brandt J T, Close S L, Iturria S J et al. Common polymorphisms of CYP2C19 and CYP2C9 affect the pharmacokinetic and pharmacodynamic response to clopidogrel but not prasugrel. J Thromb Haemost 2007; 5: 2429-2436.
17. Ticagrelor for the treatment of actue coronary syndromes. NICE Guidance TA 236. October 2011. Available online at http://publications.nice.org.uk/ ticagrelor-for-the-treatment-of-acute-coronarysyndromes-ta236 (accessed 2 July 2014).

18. Prasugrel for the treatment of acute coronary syndromes with percutaneous coronary intervention. NICE Guidance 182. Available online at http://publications. nice.org.uk/prasugrel-for-the-treatment-of-acutecoronary-syndromes-with-percutaneous-coronaryintervention-ta182 (accessed 5 November 2014).

19. Miles, M, Huberman A. Qualitative data analysis: an expanded sourcebook. 2nd ed. London: Sage, 1994.

20. North West Medicines Information Centre. Surgical management of the primary care dental patient on antiplatelet medication, 2007. Available online at http://www.ukmi.nhs.uk/med_info/documents/ Dental_Patient_on_Antiplatelet_Medication.pdf (accessed 2 July 2014).

21. Lockhart P B, Gibson J, Pond S H, Leitch J. Dental management considerations for the patient with an acquired coagulopathy. Part 1: Coagulopathies from systemic disease. Br Dent J 2003; 195: 439-445.

22. Grines C L, Bonow R O, Casey D E Jr et al. Prevention of premature discontinuation of dual anti-platelet therapy in patients with coronary artery stents: a science advisory from the American Heart Association, American College of Cardiology, Society for Cardiovascular Angiography and Interventions, American College of Surgeons, and American Dental
Association, with representation from the American College of Physicians. Circulation 2007; 115: 813-818. 23. Lillis T, Ziakas A, Koskinas K, Tsirlis A, Giannoglou G. Safety of dental extractions during uninterrupted single or dual anti-platelet treatment. Am J Cardiol 2011; 108: 964-967.

24. Napeñas J J, Hong $C H$, Brennan M T, Furney $S$ L, Fox P C, Lockhart P B. The frequency of bleeding complications after invasive dental treatment in patients receiving single and dual anti-platelet therapy. J Am Dent Assoc 2009; 140: 690-695.

25. Burger W, Chemnitius J M, Kneissl G D, Rücker G. Low-dose aspirin for secondary cardiovascular prevention - cardiovascular risks after its perioperative withdrawal versus bleeding risks with its continuation - review and meta-analysis. J Intern Med 2005; 257: 399-414.

26. Biondi-Zoccai G G, Lotrionte M, Agostoni P et al. A systematic review and meta-analysis on the hazards of discontinuing or not adhering to aspirin among 50,279 patients at risk for coronary artery disease. Eur Heart J 2006; 27: 2667-2674.

27. Collet J P, Montalescot G, Blanchet B et al. Impact of prior use or recent withdrawal of oral anti-platelet agents on acute coronary syndromes. Circulation 2004; 110: 2361-2367.

28. Gershlick $A$ H, Richardson G. Drug eluting stents. BMJ 2006; 333: 1233-1234.

29. Farb A, Boam A B. Stent thrombosis redux - The FDA perspective. N Engl J Med 2007; 365: 984-987. 